\title{
Investigating the Role of Expansive Design and Service Design in Improving Healthy Food Consumption, Case Study; Consuming Vegetables
}

\author{
Fatemeh Marzban', Alireza Ajdari ${ }^{2 *}$, Saeed Zohari Anboohi ${ }^{3}$, Yaser Pakzad $^{4}$ \\ ${ }^{\mathbf{1}}$ Ed.D. Student in Instructional Technology, Department of Curriculum and Instruction, College of Education, Texas Tech University, Lubbock, TX, \\ United States. \\ ${ }^{2}$ Assistant Professor, School of Industrial Design College of Fine Arts, University of Tehran, Iran. \\ ${ }^{3}$ Instructor, Industrial Design Department, Arts and Architecture, Islamic Azad University, Central Tehran Branch, Iran. \\ ${ }^{4}$ M.S. Student in Interdisciplinary Studies, Texas Tech University, Lubbock, TX, United States.
}

*Corresponding author: Alireza Ajdari², alireza.ajdari@ut.ac.ir

DOI: $10.22059 /$ JDT.2022.339084.1068

Received: 13 February 2022, Revised: 25 February 2022, Accepted: 25 February 2022.

\begin{abstract}
A
bstract

The goal of this study is to generate designedly solutions for improving the culture of healthy food consumption in Iran, through activity theory framework. Even though individuals might make conscious decisions to consume healthy food, their environment might prevent them from doing so. Given the current lack of attention toward a holistic viewpoint, therapy procedures as well as general healthy nutrition policies have been made useless. Observations in case study showed that social, cultural and psychological factors do have an important role in generating obesity, and the disregard for considering such factors have resulted in the divergence of patients between health centers, sports clubs, and monitoring groups. The hypothesis generated from such observations would signify that considering cultural-social context and generating a useful model, would help the patient reach a successful goal. Expansive design was chosen as the design approach in order to emphasize continuing the relationship between users and providers of service, even after obtaining the service by a user. This viewpoint and design resulted in the dialogue between the user and provider of the service or product. Based on the research, solution scenarios were generated and the third scenario, designing a collective treatment center was chosen and conceptualized through tools such as a system map, interaction story board, and consumer satisfaction diagram. The value of such a study is based on presentation and institutionalizing the theoretical infrastructures in the area of service design, while diverse solutions would be presented to specialists based on the scenario-based design as well.
\end{abstract}

\section{$\mathbf{K}$} eywords

Service Design, Activity Theory, Expansive Design. 


\section{Introduction}

Nowadays, people have access to a variety of restaurants and supermarkets, and a wide range of food choices. The process of industrialization and organization of human societies has improved the lifestyle of the modern human and enabled them to produce and consume diverse food products. Choosing the appropriate food would not only have undeniable effects on people's health, but according to Bourdieu (1986), the way of choosing and consuming food also delineates the social class of consumers (Vadadhir $\&$ Sadati, 2011). However, the increasing level of obesity in the whole world suggests the inefficacy of modern nutrition. As a matter of fact, the outbreak of nutrition-related health issues has increased dramatically, and obesity is the most problematic issue that can lead to other illnesses such as diabetes, blood pressure, chronic diseases, cancers, and others.

\section{Definition of Problem and Theoretical Concepts}

Regarding the statistics of the Iranian Ministry of Health, 50\% of casualties are because of obesity, heart attack, aneurisms, and related diseases (JahanNews, 2015) and according to a Tehran Times report on December 23, 2018, Obesity and overweight have affected about 65 percent of the country's population. More than $80 \%$ of noncontagious illnesses mentioned above have exceeded with the increasing rate of fast food and ready-made food consumption, increasing cooking oil consumption, sugary drinks, processed snacks, and advertisements for fast and processed foods. That, along with people's decreased time to eat and cook healthier foods during a typical day, also has troubling consequences on consumers' health (Young Journalists Club, 2012). The above issues can be decreased with appropriate nutrition education and medical intervention.

On the other hand, medical intervention can reduce the number of times patients visit the doctor as well as the mortality rate. Through the promotion of healthy nutrition, education, and medical intervention, we can reduce the load of treatment costs and doctor visits. The emotional costs and psychological side effects of obesity can also decline. However, there has been a lack of enough attention on the subject (Khabaronline, 2009). That is why society has witnessed an increase in medical treatment costs, psychological issues, and mortality rates associated with obesity, with no acceptance of any responsibility from the institutions or people involved. Throughout the study, which has investigated the consumption patterns of fast foods and other related causes of obesity in Iran, researchers found a meaningful relationship between people's economic situations (high income), the inhabitants' location, and the higher consumption of fast foods (Yarmohammadi et al., 2012; Ghafari et al., 2012). These results show that in societies with a high income, the level of consumption of traditional and domestic foods has decreased and these foods were replaced by fast food. Although these studies show a direct relationship between consumption of fast food in a higher income society, observation data show that people with low income in society have also consumed fast food products without considering the nutrition values because the food is inexpensive. On the other hand, people in societies with low incomes are not able to pay higher costs for their health, which consists of going to experienced doctors, high-quality treatment centers, fitness clubs, etc.

Past studies and research in Iran that concentrated on nutrition problems have insisted and emphasized on the importance of education on healthy nutrition. Different studies based on intervention programs in families show that the generation of educational nutrition intervention is needed to increase fruit and vegetable consumption and reduce obesity in families (Hashemi et al., 2012). Although educational strategies are offering the most useful methods of development and progress, they cannot solve the problem per se. This concept has been also generated based on the studies conducted by Azadbakht et al. (2003), which evaluated the awareness and attitudes of people in the field of nutrition and showed that people (in general) know about the consequences of their nutrition habits. However, the functional and real behavior of people are not just based on nutritional consciousness, and there is a gap between consciousness and real action (Azadbakht et al., 2003). 
Therefore, in the previous study, Azad Bakht has argued that, besides considering consciousness, an appropriate inter-relational nutrition program should be created and evaluated.

In order to understand more, we need to have a brief review of concepts such as obesity, health, society, and cultural and social factors.

Obesity is defined as additional body fat that has gathered. Through the negative effects of this situation, it could reduce the lifespan and increase health problems. Obesity is one of the most preventable causes of death in the whole world. The World Health Organization (WHO) has predicted that it would be possible that obesity and being overweight will cause greater health problems across the world.

Today, the discussion about health is not just about medical or biological issues, but it is quite multidimensional and consists of different physical, psychological, and social factors. Today, healthiness itself is a product or by-product of social phenomenon, and designing a program for increasing health without thinking about social and cultural aspects would not be useful (Vadadhir \& Sadati, 2011). Researchers claim that, nowadays, healthiness should not only be understood from medical or physical treatment; however, scholars should also consider the cultural and social conditions of healthiness.

In order to understand the societal and socio-cultural causes of the current situation, the researchers have used the framework for determinants of physical activity and eating behavior, developed by Booth et al. (2001). In one of these layers of the framework, Booth et al. (2001) consider the ability to choose factors such as social tendencies, situations, welfare, access to fitness facilities, and whether physical or social conditions have been studied with cost, time, security, and knowledge. The researchers will consider all of these factors to analyze the function of the target group and think about the target group's lifestyle. Attempting to improve treatment with healthy nutrition while not considering any interactive system between the potential users of healthy nutrition and the target society would decrease the effect of the treatment procedure, whether by diet or strategies of improving the health of a food culture.

The case study of this research was conducted on patients suffering from obesity. The previous observations showed a lack of success of patients although they were enrolled in a nutritional program and saw a nutrition specialist. All these observations would be interpreted as a result that unhealthy nutrition behavior and the existence of weight gain cannot be improved or changed merely by the variable of controlling calories and reducing them. Scholars also need to consider the social, cultural, and psychological factors of obesity. Given the lack of studies on these factors, patients wander hopelessly between treatment centers, fitness clubs, families, and other monitoring groups with mixed weight loss results. The primary hypothesis shows that emphasizing psychological and social contexts and modeling that in parallel with treatment procedures can have a successful result in nutrition programs. In the current case study, the researchers observed nutrition clinics in Tehran, Iran, for three weeks in 2013. The methodology for observations used a cultural and historical framework (Activity Theory) to study Iranian nutrition clinics. The present study found weaknesses in Iranian nutrition clinics; therefore, the researchers will discuss them at length. Then, the researchers will generate alternatives for improving the clinics through an expansive design and service design approach.

\section{A Critical Review of Case Studies and a Description of Possible Innovation}

Based on the researchers' observations of Iranian obesity treatment centers, nutrition clinics are currently less interested in solving the contextual problems of obesity - social, cultural, etc.-. Instead, they are more interested in obesity treatment through clinical methods - calorie counting, dieting, surgical intervention, etc.- . The researchers studied some of the most popular nutrition clinics in Tehran, such as Novin Nutrition Treatment, Baran Nutrition Clinic, Kimia Clinic, Iranian Clinic, Professional Clinic of Rasol Akram, Professional Clinic of Cibita, and Dr. Kermani's Nutrition Clinic. Among them, Dr. Kermani's Clinic was chosen because he is the most famous nutrition specialist in Iran. After that, Cibita Clinic was studied because it has a different treatment program that focuses on the contextual issues of obesity. 
Based on such studies, a general scenario was generated for further investigation, and its feasibility and applicability would be realized based on service design concepts.

\section{Analysis of Case Studies}

All the nutrition diet centers in Tehran claim that they would generate diets based on the habits, lifestyle of the person, and daily and weekly foods which exist in the family's diet. However, Cohen, Scribner, and Farley (Cohen et al., 2000) offered a structural model and suggested that the healthy behaviors would be affected by the personal situation and the condition of people based on these parameters:

1. Accessibility of protective or distractive items and products for the consumer.

2. The physical structure or the physical specification of the products.

3. The social structure and all the general politics.

4. Combination of media messages with cultural associations.

In reality, the activity of the people is based on a socio-cultural structure, in the physical structure of the society, and in the dialectic structure of their lifestyle. The role of government would be also very important. Based on these, the physical structure of the society could be structured in a way that, in every area of a street, one could see items and advertisements for fast foods, restaurants, and food courts. Out of supermarkets, one could see various snacks which are available quite easily; this has a very negative effect on children.

The existence of big markets in Tehran, like hypermarkets or welfare markets - i.e., a Walmart-like superstore-, makes available unhealthy nutritional products for the consumers. Across Iran, a lack of regulating supervision on advertisements for fast foods, junk foods, and restaurants in different forms of media currently exists. Given that such advertisements promote unhealthy food items, they can negatively affect consumers' eating habits (Amini et al., 2014; Hajizadehoghaz et al., 2016). On the other hand, the lack of time for people who have to prepare healthy food, the high cost of purchasing healthy food, and the lack of informative resources for healthy eating habits are factors that affect individual behaviors. Taken together, these variables can affect individual behaviors and prevent patients from being successful at weight loss.

Nutritionists primarily focus on the calculation and counting of calories and recommend what should be eaten and how much patients should eat. However, the same patient likely sees distractive advertisements about fast foods. Such persuasive advertisements can influence social behavior and eating habits. For example, celebrity endorsements or film/TV tie-ins in magazines and on television can influence consumers to purchase certain items. In Iran, the prevalence of fast-food advertisements has targeted young children and perpetuated the consumption of unhealthy food in society. In order to change this behavior of consumption, the current research provides prerequisites that should exist in society, not just educationaloriented solutions to solve dietary problems. Families would be successful if healthy food be more accessible (Vadadhir \& Sadati, 2011).

As well, there is no infrastructure in Iran for exchanging information between physicians and dieticians about their patients. While every hospital and nutritional center has its own file for patients who make use of their services, physicians and nutritionists do not share that information with each other. Obese patients contend with other diseases such as osteoporosis, diabetes, or chronic diseases. Therefore, all treatment centers should share records - whether electronically or another way - for illnesses, uses of medicine, and/or surgery when treating a patient in either the hospital or nutrition center. This information exchange would reduce the duration of the treatment for all patients. As mentioned earlier, nutritionists and physicians are not culture oriented but calorie oriented, and they see health from only a medical viewpoint.

Current nutrition experts and physicians don't see health conditions based on social and cultural contexts, but the potential of those contexts can help create the best services for, and understanding of what, patients and families need to consider for healthy eating habits. 
These factors are important since there is an economic and beneficial necessity in considering the satisfaction of patients, which is important in health and understanding (Lee, 2011). However, it is obvious that real treatment is based on the experiences and understanding of the patient together with other factors, which can be helpful in order to recover, improve, or manage a disease.

In a 2011 study by Dr. Naeli and Dr. Moghimi to improve the system of providing healthcare services, they identified a lack of bringing services in order to improve health in society, a decline in the quality of service, a failure to become consumer oriented, a reduction of responsibility of the system to users, a lack of documenting health services, limited information about national health accounts, and limited use of internet/computer technology — DDS, MIS, or other electronic exchange systems-.

A reflection of Naeli and Moghimi's (2011) results can be seen in the current study of a nutrition clinic. To summarize, the researchers observed the following issues in the clinic;

- A lack of communication between clinicians and patients.

- Clinicians do not focus on the patient's lifestyle choices when treating obesity.

- A lack of communication and documentation between hospitalists and clinicians who care for the same patient.

- Nutritionists only talk about consuming vegetables in terms of losing weight, not for overall health.

- Liposuction and other operations are promoted to fix obesity but are not necessarily permanent because most of these fixes do not consider long-term health goals.

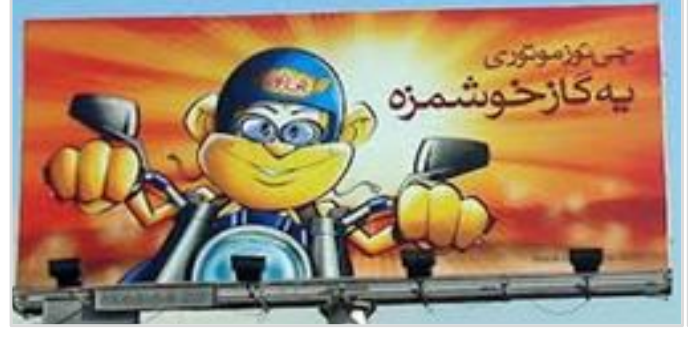

Figure 1: Advertising of Cheetoz chips, chips, unhealthy snacks (Cheetoz Motori, a sweat beat).

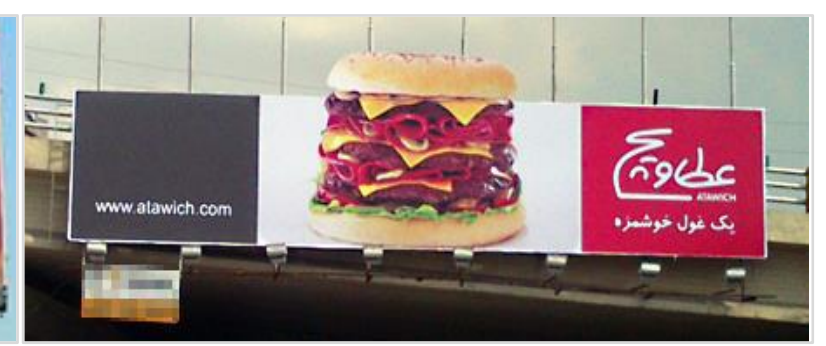

Figure 2: Atawich which is as a big sandwich (Atawich, a sweat colossus).

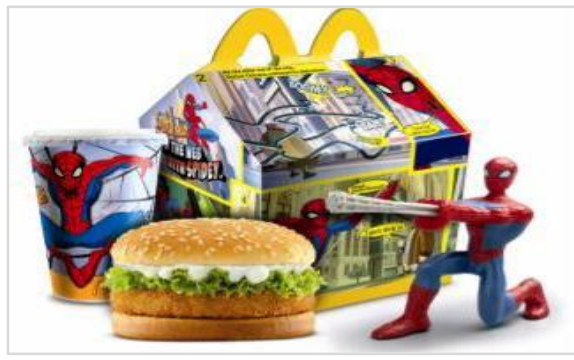

Figure 3: Happy meal of Mcdonald's.

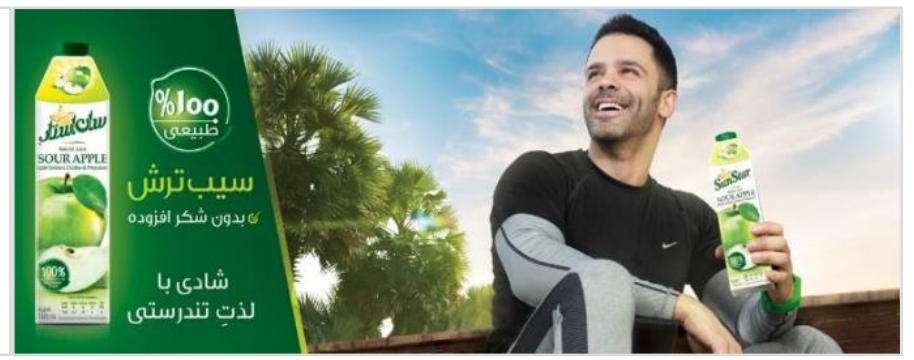

Figure 4: Advertising of SunStar (Joy with pleasure of health)

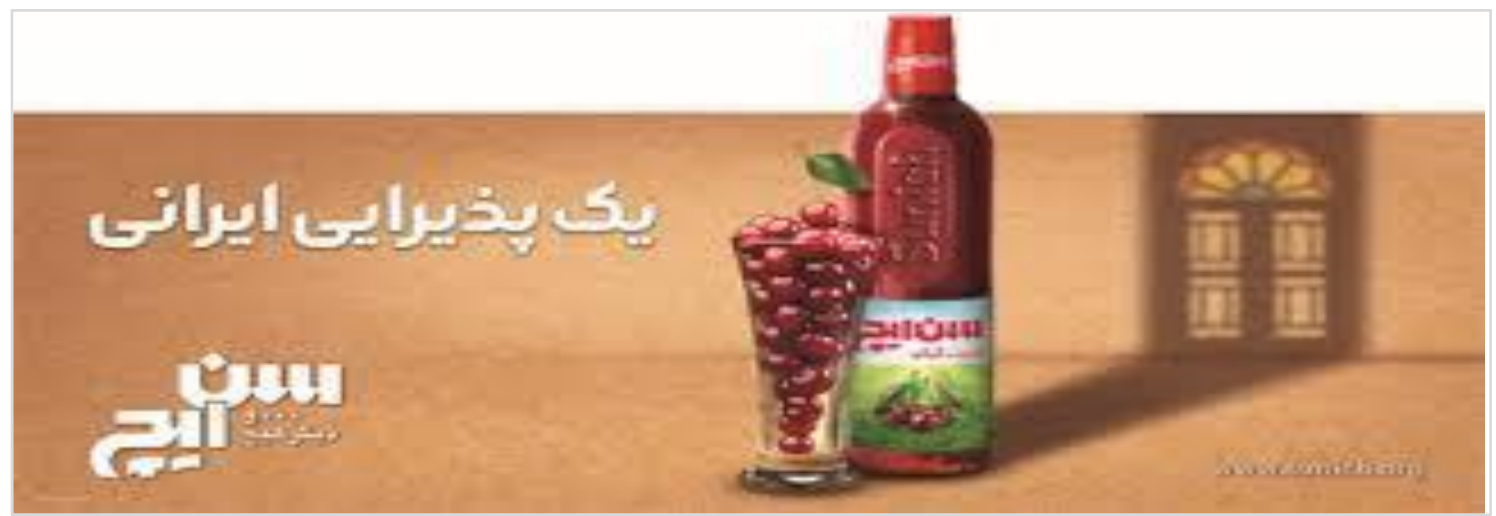

Figure 5: Advertising of Sanlch (an Iranian simple welcome's service). 


\section{Description of the Study and Theoretical Framework}

In the beginning, the researchers considered the problem with information gathered at the library and other related documents. Researchers chose the Booth model from nutrition science to analyze people's lifestyles, since this model is comprehensive. With the Booth model, these factors include social tendency, cost, time, accessibility, physical and social contexts, information resources, and knowledge with tools of ethnography such as observation and interviews. The current case studies about clinics were studied as well.

The cultural-historical Activity Theory rooted in Vygotsky psychology was used as a theoretical framework. The hypothesis of this research is that if cultural and social aspects were considered, changing behavior would be more sustainable. The researchers emphasize that human growth, based on Vygotsky psychology, is the result of a dynamic interaction between the person and society, and the character of the person is shaped by culture and society (Ormrod et al., 2016). The study has two aspects: one aspect was activity theory, which insists that every action or activity in its own context should be studied. The context is considered historical evolution and social interaction. Activity Theory has two models in design, Engström Triangle Model consisting of Subject, Object, Tool, Division of Labour, Community, and Rules; and Leontiv Model consisting of hierarchical models of operation, action, and activity - operation as physical action, action as operation plus intention, and activity as action in context-.

Activity Theory was used in this research to describe situations, model contexts, and find activity systems. Activity theory tries to theorize the contradiction in the system and show the relationship between the mixed information systems in a large context, and classifying information to reach a model of cultural complexity, describing situations, and modeling the context (Cassens \& Kofod-Petersen, 2006). As well, one of the common theories for analyzing qualitative data is Activity Theory (Bandara, 2018).

The nutrition activity and nutrition diet has been analyzed. With Activity Theory, researchers can interpret every action in its situation. Based on the Engström Triangle Model of Activity Theory, the researchers reached a model of current activities of nutrition and identified three nutrition systems;

1. Institutions of health - such as Iran's Ministry of Health, mass media, or the educational system-

2. The providers of health services — such as clinics, hospitals, nutrition clinics, and nutrition medical doctors-

3. The patients' families.

Therefore, the researchers analyzed these three parts of the system and identified contradictions which were classified into five parts: affordance gap, advertisements gap, clinic gap, educational gap, and preparation gap. Based on the three-layered model of Leontiev, the researchers analyzed the diet programs of the clinics. The Leontiev model tries to describe the behavior of the context.

There are many good examples that would show how the people would react. In fact, it shows that situations such as separation from family, stress from Konkor comprehensive exams, or even cultural situations affect unhealthy nutrition behavior. They are conditions and examples that, based on the analysis of gaps in the system, came up with three scenarios for solving the problems. These included the promotion of healthy food through schools, advertisements strategies, and clinic reform. The researchers concluded that although the biggest gap exists inside clinics, unhealthy eating behaviors and obesity would not be solved by simply controlling predefined and measurable variables, such as calorie intake and reducing it. There exist social, cultural, and psychological factors that affect the way consumers interact with their own nutrition environment. From the whole context, three factors were summarized such as the way the target users cook, access to medical care and follow medical advice, and the food consumption patterns. Shifting from a mere scientific viewpoint in clinics for nutrition behavior is a critical point and, together with cultural and social situations, the current study hypothesizes a more successful diet program. The first step in the current study's design begins at a nutrition clinic where design tools, such as system map, interactive storyboard, and customer satisfaction diagram would be implemented.

Activity theory's job is to criticize the current culture and generate an alternative culture; however, generating culture is not just in the domain of food but also in the consumption of food. 
There are many conditions and situations in which unhealthy nutrition behaviors are advertised - see Figures 1-5_. These are just a few instances of mottos or slogans that try to detect cultural conditions and situations and generate a culture for consumable goods. Other advertising visuals and psychological elements used in advertisements can help researchers understand the problem better. In this condition, consuming a product generates its own culture. Fast food has its acculturation process, but what about healthy food? There is almost no positive activity in this direction. Another way to reach a better result is to check the history of fast food consumption and discover why such behavior was generated. This is not something that could be analyzed just through calorie intake but would be discussed in conditions and situations such as stress eating or smoking.

On the other hand, the second part of the work needs to use expansive design because of the lack of relationship between the patient and the doctor. There is no network, no unification of information, and the people themselves should carry their medical records to the other doctor. In another word, doctors and diverse monitoring agencies would not be connected to each other. In other words, doctors and diverse monitoring agencies are not connected. As a result, the beneficial and comprehensive information would not be sent or transferred to doctors to bring out the patient's problem. An expansive design approach with its elements would address this gap. This is something that is operationalized through dialogue-making tools - care maps, care calendars, and care agreements. On the other hand, in the market, there is competition between nutrition centers to offer distinguishable services to absorb patients. This would be important about what kind of experience patients and families have in their facilities or how people and patients would reach the quality of a healthy situation. It is important how users would understand the quality of health in therapeutic environments (Lee, 2011). In Iran, there is no attention paid to the services for patients. Research conducted in 2014 about services in health centers showed that the services given are in no way beyond the expectations of the users and there is a lot of space for improving services (Safi et al., 2014). Based on the studies and based on the concepts given in the design of a new model, social factors have been used and conditions have been added to social and cultural aspects, which can improve health conditions. The below model illustrates the pattern of improving patient health when considering social and cultural conditions.

\section{Proposed Model}

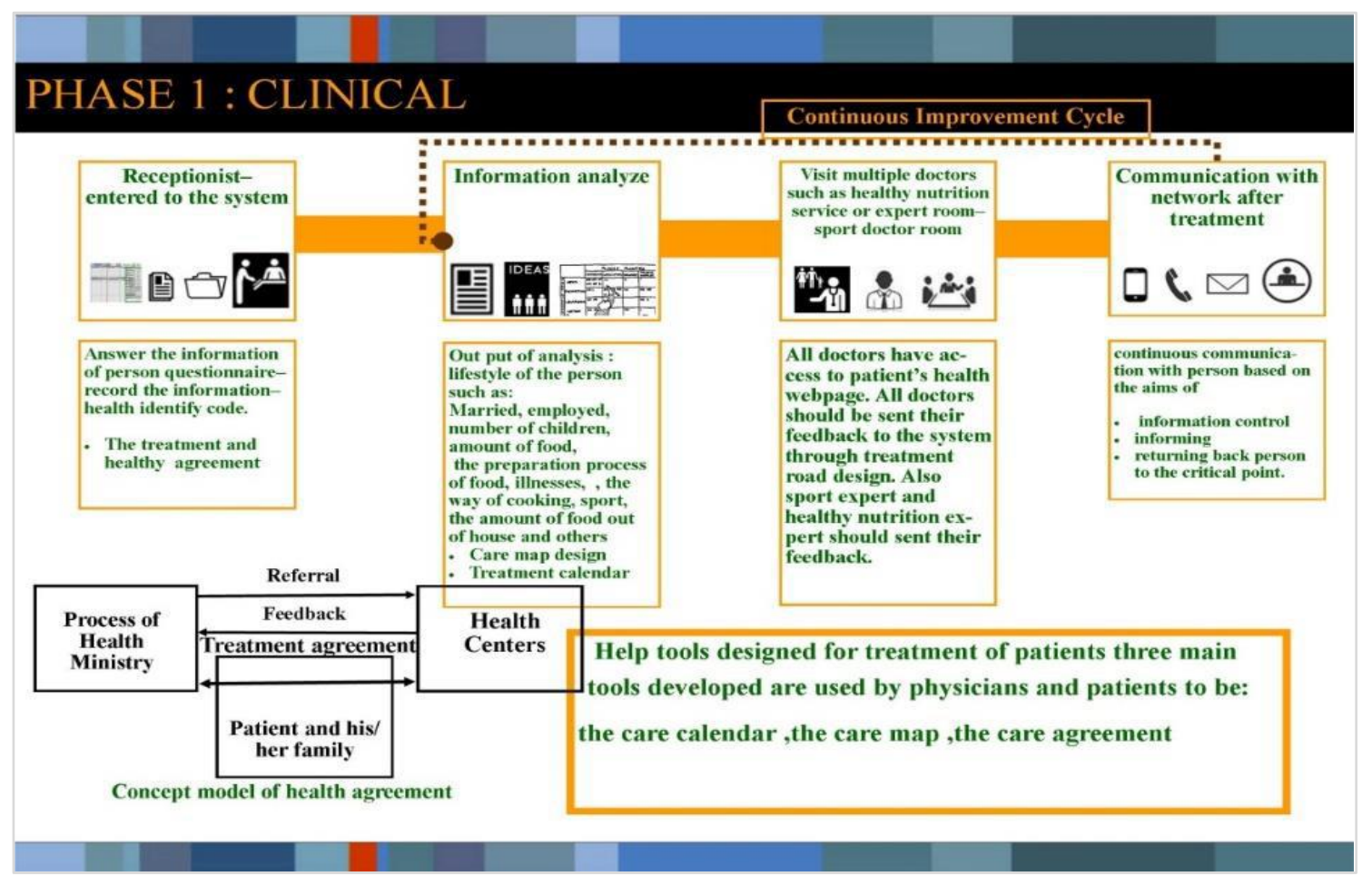



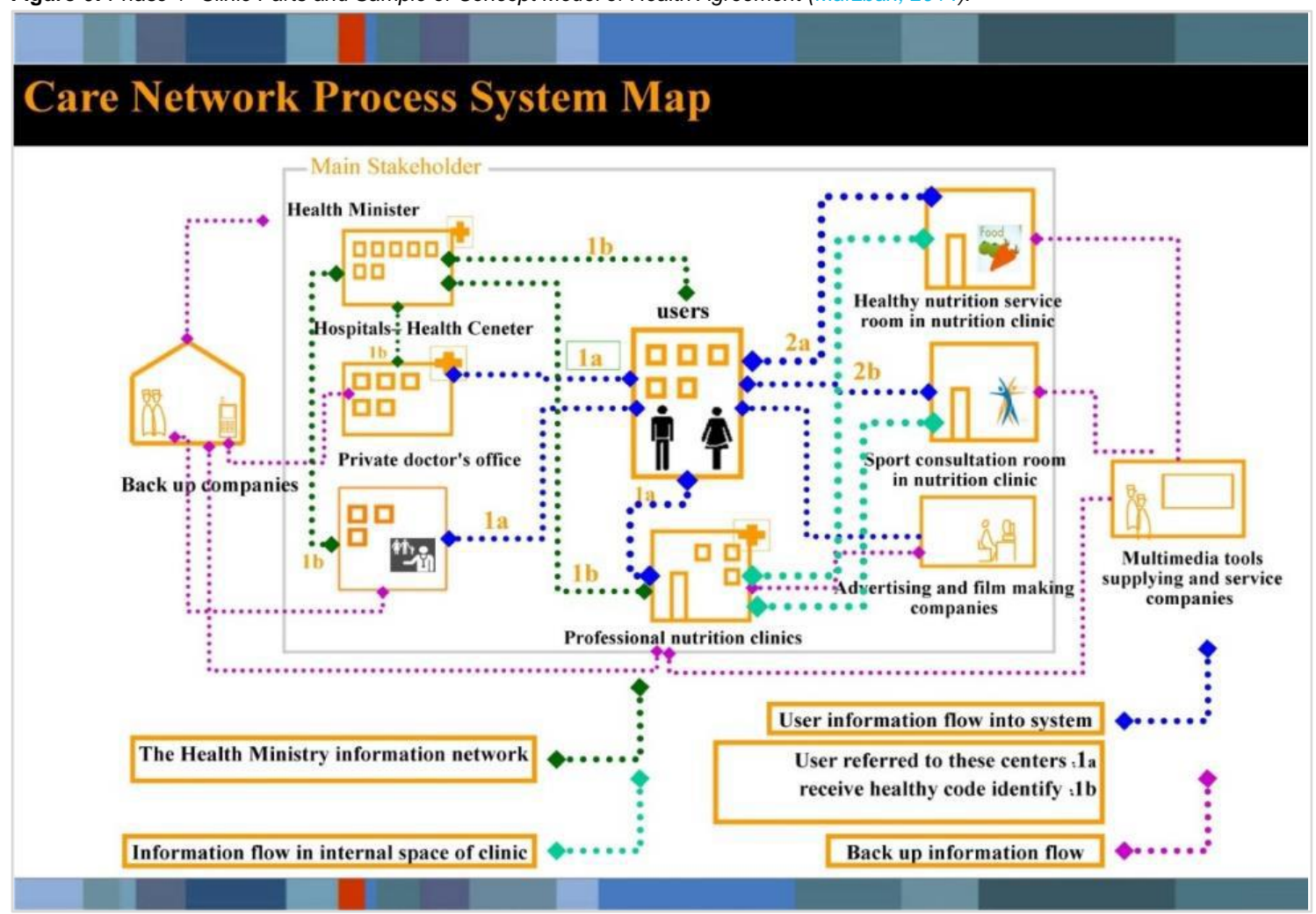

Figure 6: Care Network Process System Map- Information Flow (Marzban, 2014).

As the hypothesis of this study was taken into account previously, that is if cultural and social aspects were considered, changing behaviors would be more sustainable, this is considered in the context of the nutrition clinic. This model aims at generating unification of information. There is, of course, a necessity for electronic infrastructure. In the first place, the patient would enter a health electronic system based on the operators and they would be given a health code. Information exchange would be electronically offered to all systems of the clinic. Regarding the health system, all the clinical operators such as nutritionists, psychologists, nutrition experts, sport experts, or sport doctors, would have access to the webpage of information about the patient's health. These operators are expected to take care of the treatment and would have to submit every consultation into the system. So, the patient would enter personal information after being registered in the health center's electronic system, and this would be analyzed by professional experts in psychology and other nutrition assistants. Therefore, in this phase, based on information of the patient, dialogues would start and a care map would be generated. All the information that nutritionists find would be entered in the care map and care calendar ${ }^{1}$.

Another aspect of the new model's health program is consultations for healthy nutrition along with cooking services with private chefs. Food preparation for healthy eating and vegetable consumption play an important role in nutrition. The other part of the treatment includes working with a sports professional as

1 Patient's marital status, employment status, the kind of job and the conditions regarding job and the conditions regarding stress, social activity, socia support, how they deal with their problems, family situations, any problematic behavior such as medicine consumption without prescription, lack of attention to medical recommendations, the amount of food they eat, the kind of food they eat, the amount of food they eat out, the amount of fast food they eat, what sports they play, how they prepare and cook food, and they have any illnesses. Emotional well-being and the frequency of vegetable consumption would be also important factors. well as a medical advisor. Their task is to bring activity back to the life of the patient. The information given to the nutritionist in general terms would be defined by details. The patient is completely exposed to environmental ads while staying at the clinic. Advertising agencies and filmmaking companies provide the patients with video clips about different uses for vegetables under their doctor's supervision. Moreover, current and former patients who are interested in sharing their very personal experiences with others are also welcome. The sport and food consumption rooms that exist in the internal network of the clinic will enter feedback information. The clinic will have someone analyze the saves for further communication with the patients after they complete the program. 
The system map extracted from the Cibita model was chosen; however, some other elements were added, as mentioned below:

1. The agency of healthy nutrition service: since patients cannot teach themselves how to cook and the correct way of food preparation, they need to communicate with cooking experts, who will help them learn how to cook vegetable-forward foods. So, experts would explain it to patients and answer all their questions. Based on the current situation of nutrition clinics, they do not consider the reasons why patients do not consume vegetables, and they do not come to know about different contexts that can explain a patient's behavior. However, in the new model, the section of healthy nutrition services can be used to fill this gap. Cooking experts can ask patients about which types and tastes of vegetables are not of interest to them. Moreover, experts can teach patients new recipes, cook food for them to sample, and instruct patients on how to prepare vegetables in ways that taste good. This information will also be recorded so that a database is established to help and improve the research and development. The experts can reach an understanding of patient perspective, especially those who are advised to eat more vegetables, but take that advice for granted. These verbal protocols can be recorded such as, I don't like cooked vegetables or I just look at vegetables as medicine or having medical effects. This way, by trying different tastes, the expert can encourage the individual to use vegetables and learn about the reasons why patients do not cook vegetables.

2. The agency of data analysis: After patients leave the clinic, specialists with access to the patient's information - including illnesses and nutrition habits - will stay in contact with the patient and continue to help them on their path to a healthy life. And when a person moves away from the arranged schedule, they will be helped in coordination with other parts of the clinic. So, in reality, a continuous effective relationship between patients and the clinic would be shaped in order to implement the communication with the patient and return them to treatment.

3. Agency of advertising and filmmaking clips: Agent of advertising and filmmaking will make clips related to the health and consumption of vegetables. People who are willing to share their experiences with others can work with the agency of advertising and filmmaking under the supervision of their doctor, and show the film to other patients in the clinic. The advertising companies have the role of advertising in accordance with the clinic's management, and they can even make films about those who, in current treatment for a healthier life, suffer from both obesity and other illnesses. These films would be shown in the clinic. In fact, new ideas from the films could help patients in this way. In reality, all patients would be surrounded by the clinic's advertisements that they consume healthy food.

4. Agency of support system: software and related applications would be designed for clinics connecting it with the profiles of patients stored in electronic health services. All parts should have access to the networks.

The method used for the service designs was called MEPSS or Methodology for Product-Service System development. These are introduced by Prof. Carlo Vezzoli, and it refers to the tools such as system map, interactive storyboard, and consumer satisfaction diagram. They would help us to have products and services together with the demand of consumer satisfaction. The synergetic effect would be multiplied for multi-layered intervention. On the other hand, in consideration of expansive design, three tools create a dialogue and unify information: the care calendar, the care map, and the care agreement. For this method, the tools will be: treatment calendar, treatment map, and treatment agreement.

Treatment calendar consists of a simple pattern to summarize the happenings during the patient's treatment and procedures of treatment. The structure of the treatment calendar needs a bilateral conversation between the doctor and the patients. In other words, the treatment calendar would be based on the co-configuration in expansive design related to the expansion of time (Engeström, 2006). The treatment map is a one-page format for graphical presentation of the nurses and other institutions that are engaged in the patient's treatment as well. In an ideal situation, the doctor and the patient together would generate the first version of the map's structure; the relationship between the doctor(s) and a patient would be included on the map. So, the care map can identify and detect the gaps and separations in the care unit or system. The care map can also expand social and local parts of the expansive design (Engeström, 2006). 
A care (treatment) agreement is a one-page format that needs the doctors and the patients to show, detect and record concerns of the patients (Engeström, 2006). It also includes work distribution in treatment, the methods of informing each other, the date that the care agreement would be assessed again, and signatures from different people involved in treatment. Generating a preposition of care agreement needs exchange and negotiation between the patients and people involved in their treatment. The need for negotiation or repeated negotiation is the responsibility of the care agreement and ethical expansion from the ideological and systematic viewpoint which should be both considered. The goal is to improve the person's lifestyle. In this set together with nutritionist experts, there is a psychologist to understand the lifestyle, an expert in healthy nutrition, and a sports expert who will give the needed guidelines to the users. This set of guidelines includes the improvement of the lifestyle of the whole network in order to communicate and improve relations between the set and the user even after leaving the program. So, the tools of the dialogue consist of care map, care calendar, and care model.

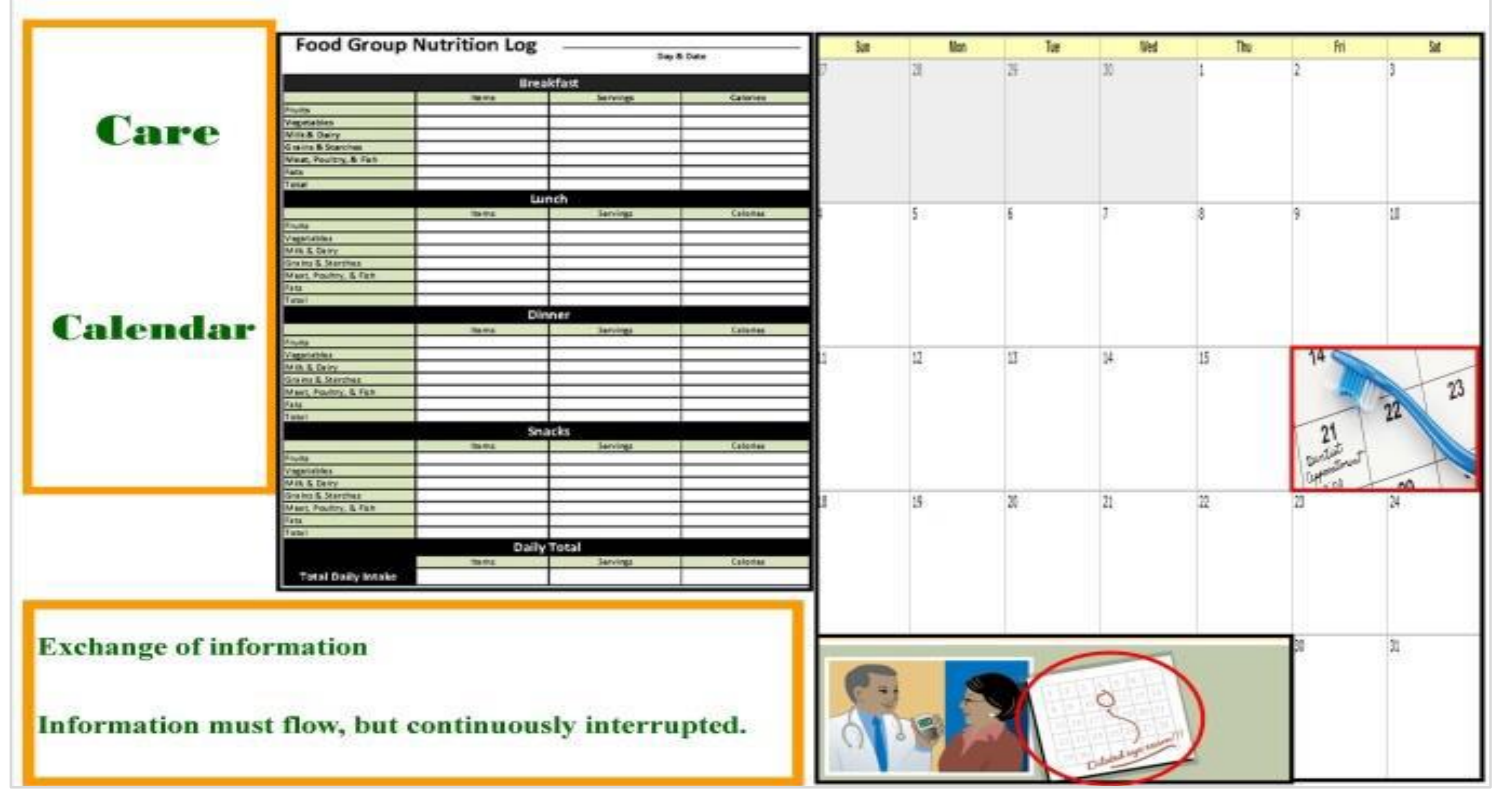

Figure 7: Treatment Map (http://www.aafp.orgfpm20030400p33.htm/).

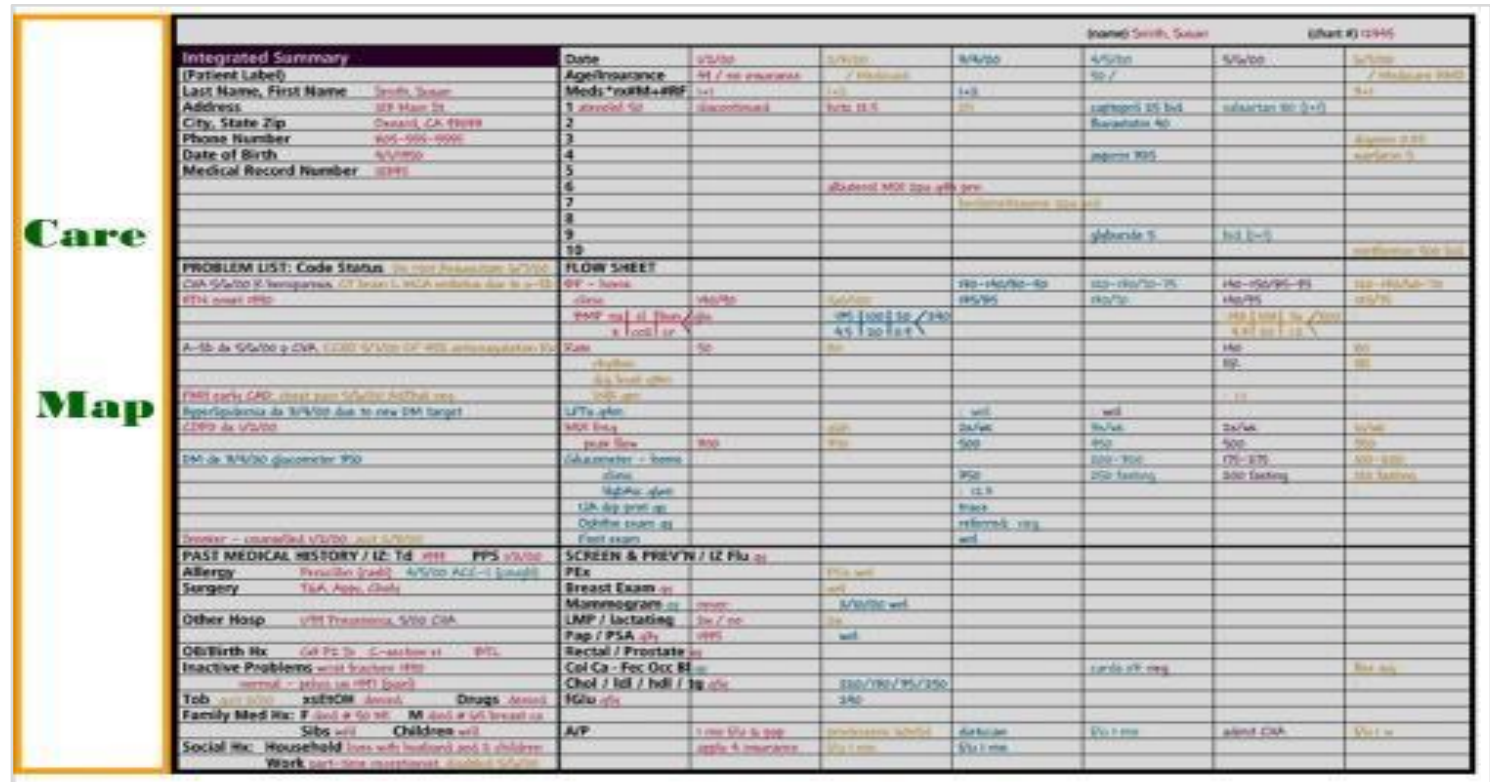

Figure 8: Treatment Calendar (https://www.hashdoc.com/documents/5991/food-group-nutrition-log). 


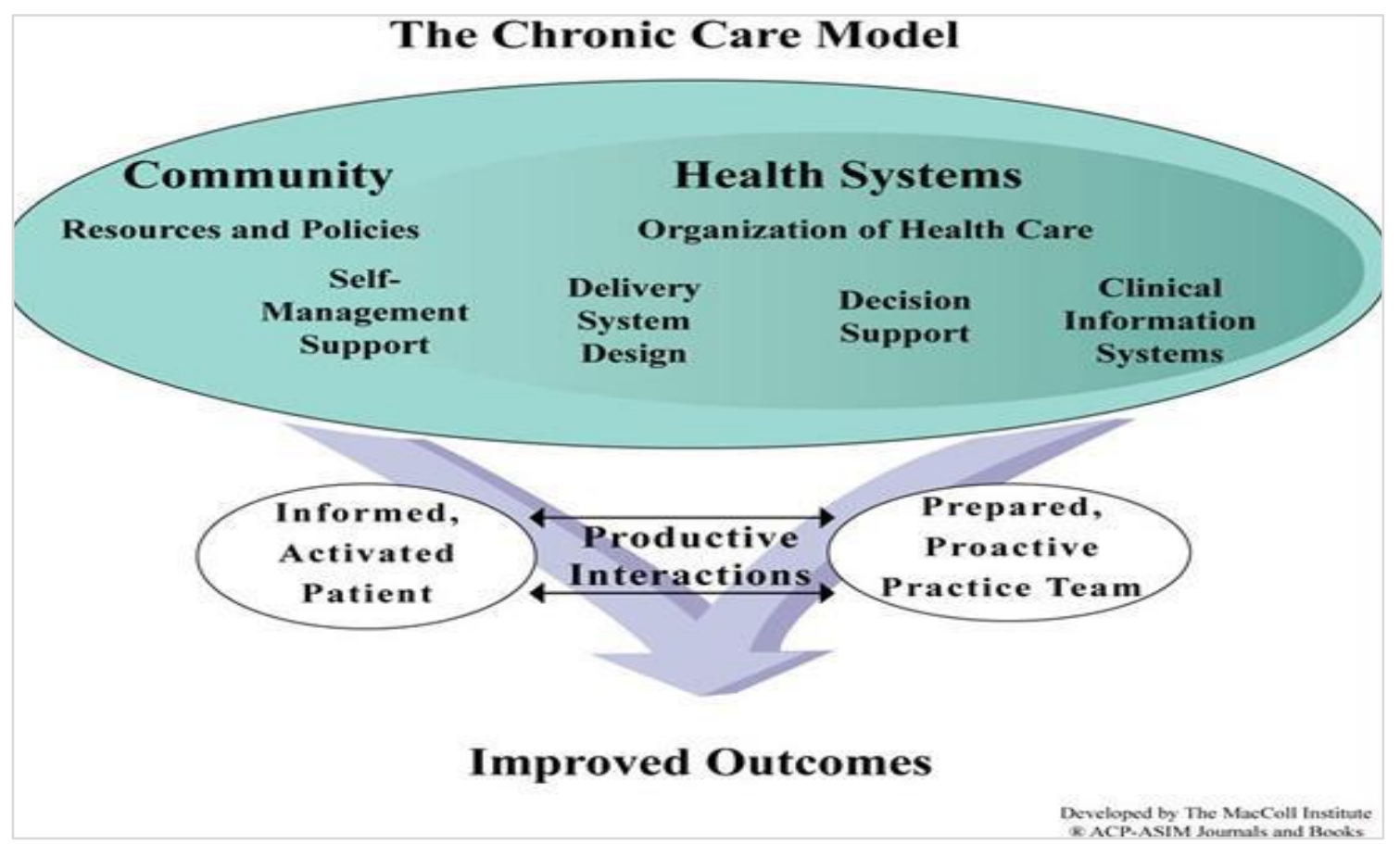

Figure 9: Concept Model of Health Agreement (http://www.improvingchroniccare.org website).

\section{Test and Validation}

After presenting the model, the researchers designed a new model in order to validate and see the correction of the model. The researchers needed some references in order to do the validation because they did not have enough equipment and facilities to perform and build the model. They interviewed two nutritionist experts: Dr. Shokravi and Dr. Zaker (Cibita principal) and explained the model to them. The Cibita Clinic is a very good reference for testing the other features that were added to the Cibita model, such as the healthy nutrition service and information analyzer advertisement, filmmaking companies - designing the environmental advertisement-, and the backup network. The generated questions for those two experts are:

- From your point of view, could parts of the healthy nutrition service model improve the health and treatment of obese patients and increase vegetable consumption?

- Regarding your viewpoint, what parts of the analyzer, which is designed to understand, would make continuous contact with the patients, and could it have an effect on a procedure?

- From your viewpoint, how can filmmaking and advertisements affect the viewpoint of the people about consuming vegetables and having a treatment procedure?

- Why didn't you agree with the diets introduced by a nutritionist?

- How could the tools of the treatment path - treatment map, treatment calendar, and treatment agreement - be helpful for a nutrition model, and what could be the main reason?

- Could we have a better result by implementing the treatment path in Cibita's model? Could the design of the tools of the treatment path - the care map, the treatment calendar, and the treatment agreement - all be useful for the Cibita model, and why?

- Could we have a better result by generating appropriate instruments for Cibita Clinic?

- How might issues, such as the economic expansion of Cibita Clinic, be evaluated or assessed?

From Dr. Shokravi's viewpoint, the first part, which is about the nutrition information analyzer, could be very good for the patients coming to the clinic for the first time, so nutritionist experts could have better knowledge about the patients. It is more economical and appropriate, but it could not be very useful for users coming for a second or third time because the number of patient visits would be less. Since qualitative work is needed to be done, the person involved should also be involved in benefits. 
As a result, the price and the costs of the service would be increased, which is not good. She also added that after the fourth session, the controlling of the information would be done by the nutritionist themselves. Hence, it is very good for the first-time visit, and the psychologists could extract the basic information and improve it. Concerning healthy food service, she has said that right now we are promoting healthy food nutritionist services by phone because the patients do not have time to read the diet program; and even when they read it, they cannot generate questions until they return home and think about it. In other words, when a person is interested in an action, questions come to their mind; this could be used for following sessions and solving them. At the first point, when the analyzer information was explained to Dr. Shokravi, she could not accept that because of the wrong thought that doctors typically do not call their patients. Patients must come to their doctors. After more explanation; she accepted this part can help patients come back to their treatment procedure and she mentioned that it can be useful.

She has considered all tools of information unification useful and insisted that they have such a connection with patients' doctors, especially those patients who have diabetes. However, the criticism is that all these are not instrumented and targeted. In other words, the obvious point here is that there is no, or not enough, attention paid to people's conditions and situations by nutritionist experts. Dr. Zaker was totally against giving a mere diet but insisted on the necessity of complementary parts of the approved ration service, mentioning that a helpful environment should be generated for the people so they could be satisfied with the whole model; however, he was not interested in discussing the issue further. However, the Cibita Clinic could be a good validation reference for this regarding the expenses. The viewpoint of Dr. Zaker was that if this work could be more applicable, people would like to pay and he was quite happy that this study reach such a design. Although the researchers are not nutritionists, it is just an introductory approach for this paper and the proposal was quite qualitative and further investigation should be conducted later.

\section{$\mathbf{R}_{\text {esults }}$}

Based on the current study's findings, failure to provide proper services, a lack of being consumer oriented and non-culture oriented, and the lack of implementing sharable patient information platforms among clinicians are the primary factors that prevent clinics from successfully treating patients.

The study has a hypothesis itself which proves that changing behavior based on cultural and social contexts would be more sustainable, this was generated regarding to the qualitative interviews with two nutritionists based on their expert views. The experts interviewed in this study have also described such a problem; data was generated from an interview with Dr. Zaker, the principal of Cibita Clinic, showing that the results can have equivalent results regarding the proposed hypothesis. Based on the expert analysis by Dr. Shokravi, the design would take too much time and money, and patients may not be able to pay for treatment. Given Dr. Shokravi's concerns, future research should develop a more time- and cost-effective way to implement a new program.

Some suggestions, for example, would be the development of a software program to save the time of doctors, or using the tools based on Engeström laws. Another solution is outsourcing, meaning that tasks would be divided between others, so it would be clarified which parts are conducted by physicians, and what part of the treatment process could be done either by the families or through consultation. Another suggestion is working on persuasive techniques and persuasive affordance based on the users, so something is needed to be done in order to motivate the users. If researchers could trace this problem and better understand all the pre- and post-conditions affecting patient health, then, based on that fruitful work, they could create a better acculturation process (mottoes or slogans) that emphasizes healthy nutrition for patients every day during and after treatment. These would generate a more sustainable change. Regarding these studies, clinicians have to help people maintain their health while expanding a healthy nutrition system to help users change their daily habits in a more sustainable way. 
Regarding all these preconditions of behavior changes, each part of healthy nutrition would give patients a new experience and a new persuasive and pleasant sign of change. Whenever every patient thinks that they would be motivated and persuaded, they would reach a more sustainable solution. And there is a tremendous complexity behind the intentions that humans have for their actions. Some of the questions that this study cannot answer are the following:

1. Can branding be at least partially effective in the demand for healthy nutrition?

2. Would all the syntagmatic parts of the system improve Iran's health system?

3. What behavioral products can be designed that could help improve health education?

4. In order to let the users find the path to healthy food, what behavioral products can be designed?

5. Can events such as salad exhibitions help solve problems related to food consumption?

6. What are the social and cultural motivations that could help to promote healthy nutrition?

7. Can clinics serve as a motivation point of acculturation, as studied in this article?

8. Is a person's acculturation to food products similar to their acculturation to other products, and how can this design help improve a person's acculturation to food products?

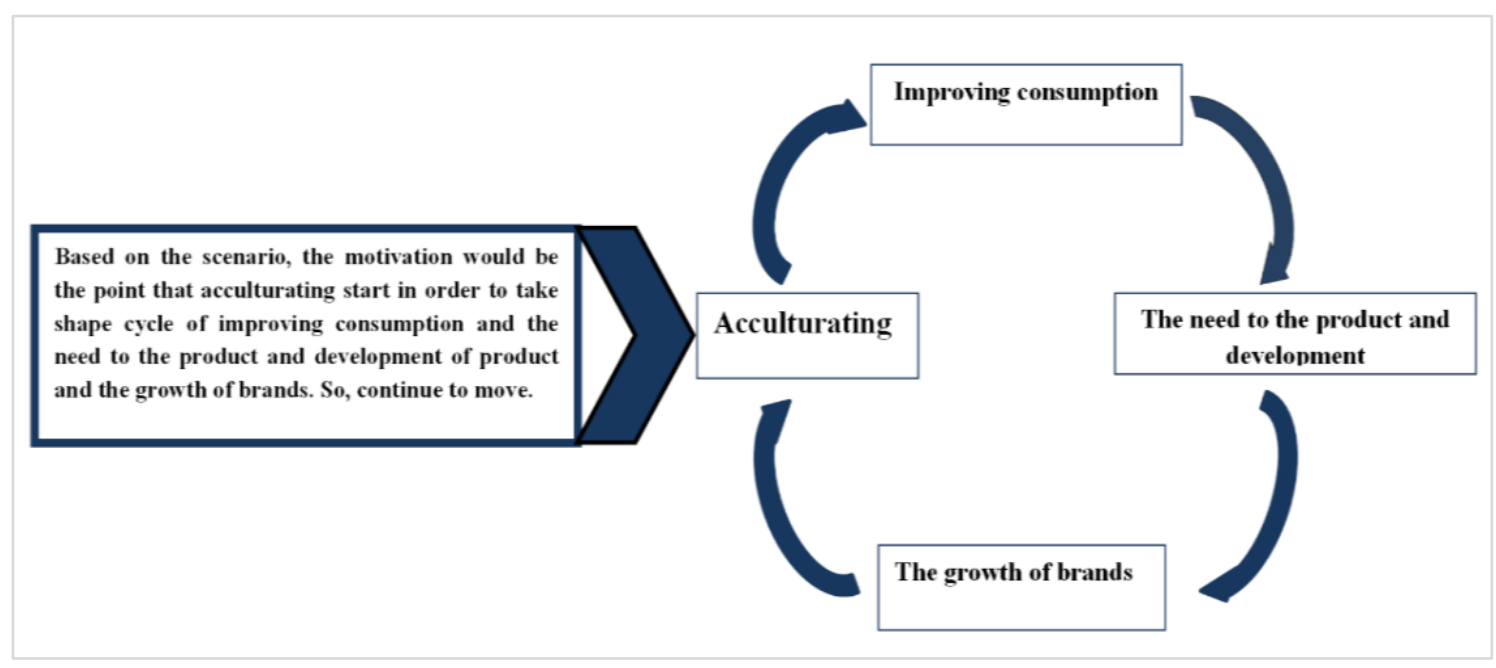

Figure 10: Improving Consumption Cycle (Marzban, 2014). Diagram illustrates the acculturation cycle of Food Products (vegetables). The cycle of increasing healthy food consumption starts from acculturating (culture), a point that contributes to the growth of demand from individuals and supply by the market. Demand creates supply. In the development of food products, demand in the market is very important. After increasing demand, product and product development is needed. Then, advertising can help grow the brands, and then, growth of brands can enhance healthy food consumption.

\section{Limitations}

Other factors that need to be considered are advertisements, attitudes of peers, ceremonies - importance of food with high fat-, social support, lifestyle, job type, location of inhabitance, income, social activity, stress, accessibility, and social class. They all affect the conditions of ways people live, the condition of work environments, working class life, psychological health, how people deal with their problems, highrisk behaviors, lack of attention toward health care recommendations, inappropriate nutrition habits, lack of attention toward accessing clinical care, lack of going on time for medical treatment, and the family situation that includes lack of family support. As these conditions have not been understood, they have all been denied or ignored thanks to the lack of attention toward the contextual conditions of Iranian society and people's lifestyles. They have an important effect on the behavior of people, and working on Iran's social environment and other surrounding institutions would have a permanent effect on nutrition behavior in the country. 


\section{Conclusion}

The purpose of this study was to look at the obesity problem from a design perspective and generate generating designedly solutions. The hypothesis of this study was that socio-cultural factors play an important role in promoting sustainable behavior.

One of the most important findings in this study was that there is a gap between people's knowledge and action. Another finding of this study was that clinics do not pay attention to the social and cultural context of patients' behavior.

A new model was designed in order to consider the socio-cultural context in nutrition clinics. The next step that designers could work on is knowing the new happenings which take place in the field of the new activity (meaning that since there are four new parts in the new model, the new cycle of activity will occur; in this regard, patients will be visited by a sports expert, cooking expert and psychologist, in addition to a nutritionist; thus, new activities will be formed) and learning from the new cycle. Based on that, designers could work on eliminating problems and improving work. From the other aspects of this study, another next step is about behavioral products for changing the behavior like applications, so patient and clinic could be connected all the time. External signs strategies for behavioral products, such as putting products in the user's daily environment, using a slightly different sign each time to avoid being ignored, making a strong and meaningful association with parts of a person's daily routine, so users can use the product to help improve and expand the new model (Wendel, 2013). Clinics can use different advertisements and products to help remind patients about changing their eating habits. Local governments can also use this strategy for cities in order to recommend that people eat healthy food. All these can help the situation and improve the model. This model can be expanded and be more compatible if clinics hire service designers to identify new activity cycles and solve the problems of the clinics.

\section{$\mathbf{R}_{\text {eferences }}$}

Azadbakht, L., Mirmiran, P., Momenan, F., \& Azizi, F. (2003). Assessment of knowledge, attitude and practice of middle and high school students in District 13 of Tehran in the field of healthy nutrition. Iranian Journal of Endocrinology \& Metabolism. 5(4), p. 409-416. [In Persian]

Amini, M., Omidvar, N., Yeatman, H., Shariat-Jafari, Sh., Eslami-Amirabadi, M., \& Zahedirad, M. (2014). Content analysis of food advertising in Iranian children's television programs. International Journal Preventive Medicine. 5(10), p. 1337-1342.

Bandara, S. (2018). Activity theory is an effective framework on qualitative data analysis in social sciences studies. International Journal of Research - Granthaalayah. 6(7), 85-95. https://doi.org/10.5281/zenodo.1323013

Booth, S. L., Sallis, J. F., Ritenbaugh, C., Hill, J. O., Birch, L. L., Frank, L. D., Glanz, K., Himmelgreen, D. A., Mudd, M., Popkin, B. M., Rickard, K. A., Jeor, S. St., \& Hays, N. P. (2001). Environmental and societal factors affect food choice and physical activity: Rationale, influences, and leverage points. Nutrition Reviews. 59(3), p. 21-39. DOI: 10.1111/j.1753-4887.2001.tb06983.x

Bourdieu, P. (1986). The forms of capital. In Richardson, J., Handbook of Theory and the Search for the Sociology of Education. Greenwood. p. 241-58.

Cassens, J., \& Kofod-Petersen, A. (2006). Using activity theory to model context awareness: A qualitative case study. American Association for Artificial Intelligence. p. 619-624.

Cohen D. A., Scribner, A., \& Farley, T. A. (2000). A structural model of health behavior: A pragmatic approach to explain and influence health behaviors at the population level. Preventive Medicine, Elsever Journal. 30(2), p. 146-154. https://doi.org/10.1006/pmed.1999.0609 
Engeström, Y. (2006). Activity theory and expansive design. In Bagnara, S., \& Crampton-Smith, G. (eds.) Theories and Practice of Interaction Design. Hillsdale: Lawrence Erlbaum. p. 3-23.

Ghafari, M., Ebadi, L., Ramazankhani, A., \& Souri, H. (2012). Fast food consumption and its related factors among students: A cross-sectional study. Journal of Health System Research. 8(6), p. 981-90. [In Persian]

Hashemi, B., Omidvar, N., Bondarianzadeh, D., Shakibazadeh, E., Rashidkhani, B., \& Abbasian, F. (2012). Effect of a family-based intervention based on social-cognitive theory on fruit and vegetable intake of middle school female students in a district of Tehran. Hakim Research Journal. 15(1), p. 44- 52. [In Persian]

Hajizadehoghaz, M., Amini, M., \& Abdollahi, A. (2016). Iranian television advertisement and children's food preferences. International Journal Preventive Medicine.7(1), p. 128. DOI: 10.4103/2008-7802.195825 Jahannews. (2015). Statistics of ministry of health about of casualties' obesity in Iran. Accessed 22 April. http://jahannews.com/vdciuva5ut1azv2.cbct.html [In Persian]

Young Journalists Club. (2012). Obesity is the basis of non-communicable-diseases. Accessed 1 November. https://www.yjc.news/00HOCu [In Persian]

Khabaronline. (2009). Tripled the number of referrals to the hospital due to obesity. Accessed 30 October. http://www.khabaronline.ir/(X(1)S(nlrtbv2qeavtvxmgxuesior2))/detail/22127/society/health [In Persian]

Tehran Times. (2018). 65\% of Iranian population overweight, obese. Accessed 23 December. https://www.tehrantimes.com/news/431000/65-of-Iranian-population-overweight-obese

Lee, S. (2011). Evaluating serviceability of healthcare servicescapes: Service design perspective. International Journal of Design. 5(2), p. 61-71.

Marzban, F. (2014). Improvement of nutrition culture using design approaches in form of presenting a collection of products (enhancement of vegetable consumption in families). Unpublished Master Thesis. Islamic Azad University. Tehran.

Naeli, J., \& Moghimi, D. (2011). Iran's primary health care promotion policy document - disease study. http://www.phcq.ir/Portals/0/Documents/Sanad-Siasat.pdf [In Persian]

Ormrod E. J., Anderman M. E., \& Anderman H. L. (2016). Educational Psychology: Developing Learners (9th edition). Pearson.

Safi, M. H., Fereydounfar, A. A., \& Arshi, S. H. (2014). Quality of primary health services in the clinics of shomal health center of Tehran. Community Health. 1(1), p. 54-61. [In Persian]

Vadadhir, A., \& Sadati, M. H. (2011). Health at social-cultural life in Tehran city. Society and Culture. Tehran. [In Persian]

Yarmohammadi, P., Sharifirad, Gh., Azadbakht, L., Morovati Sharifabad, M., \& Hassanzadeh, A. (2012). Predictors of fast food consumption among high school students based on the theory of planned behavior. Journal Health System Research. 7(4), p. 449-459. [In Persian]

Wendel, S. (2013). Designing for behavior change: Applying psychology and behavioral economics. O’Reilly Media. 